\title{
Progestogens and breast cancer risk: the role of hormonal contraceptives and hormone replacement therapy
}

Jo Marsden, MD, FRCS (Gen Surg), Senior Clinical Fellow, Department of Academic Oncology, Hedley Atkins Unit, Guy's and St Thomas's Hospital NHS Trust, London, UK, Roger A'Hern, Department of Computing and Statistics, The Royal Marsden Hospital NHS Trust, London, UK

Correspondence: Miss Jo Marsden, Department of Academic Oncology, Hedley Atkins Unit, Guy's and St Thomas's Hospital NHS Trust, St Thomas Street, London SE1 9RT, UK. Tel: +44 (0) 2079555000 ext 3321. Fax: +44 (0) 2079558730.

E-mail: jo-marsden@yahoo.com

(Accepted 15 August 2003)

Journal of Family Planning and Reproductive Health Care 2003; 29(4): 185-187

Most breast cancers are oestrogen-dependent. In addition, large randomised trials have demonstrated improved survival for women with hormone-sensitive disease given treatment that reduces the synthesis or activity of endogenous oestrogen. ${ }^{1}$ This level of clinical evidence, and the fact that early menarche and delayed menopause are risk factors for breast cancer, support the hypothesis that exposure to exogenous oestrogen will increase the risk and mortality from oestrogen receptor-positive (ER+ve) breast cancer. By contrast, recent publication of the placebocontrolled, randomised Women's Health Initiative (WHI) study and the observational Million Women Study has focused attention on the role of exogenous progestogens in the development of postmenopausal breast cancer. Inevitably the effect of contraceptive progestogens, used alone or combined with oestrogen, has also come under scrutiny.

\section{HRT and breast cancer: evidence from the WHI and the Million Women studies}

In June 2002, interim analysis of the WHI study revealed an excess of breast cancer events in women allocated to continuous combined hormone replacement therapy (HRT) [i.e. conjugated equine oestrogen (CEE) $0.625 \mathrm{mg}$ plus medroxyprogesterone acetate (MPA) $2.5 \mathrm{mg} .^{2}$ As a result the combined HRT arm of the WHI study was closed prematurely. No similar increase in breast cancer was found in the unopposed oestrogen arm of the WHI study, which remains open. ${ }^{3}$ Subsequently, the WHI study investigators have published details of the impact of combined HRT on tumour incidence, phenotype and mammography. ${ }^{4}$ With a mean follow-up of 5.6 years (estimated mean duration of HRT use of 3.1 years) the odds ratio for invasive breast cancer was 1.24 (95\% CI 1.01-1.54), which is similar to estimates from prior observational studies. ${ }^{5}$ In absolute terms, the event rate per 1000 women using combined HRT is four extra invasive cancers with 5 years' use; an excess risk of 1 in 250 . The estimated odds ratio for 2 years' exposure is not significantly elevated $(0.72 ; 95 \%$ CI $0.47-1.10)$. There was no evidence for interaction with other known breast cancer risk factors.

The Million Women Study collected questionnairebased data on lifestyle, socio-economic background, reproductive and medical history and HRT use from a total of 1084110 women aged between 50 and 64 years who accepted an invitation to attend the National Health Service Breast Screening Programme (NHSBSP) between 1996 and $2001 .^{5}$ One-third of the women were current users of HRT at the time of questionnaire completion. ${ }^{6}$ With an average follow-up of 2.6 years, 9364 breast cancers were diagnosed. The mean time between questionnaire completion/baseline mammography and cancer diagnosis was 1.2 years. Only current HRT users (mean duration of 5.8 years) had an increased breast cancer risk [relative risk (RR) 1.66 ; 95\% CI 1.58-1.75] and there was evidence of a 'duration of use' effect. Risk was increased with all types of HRT preparations including tibolone but the greatest risk was conferred with combined HRT, irrespective of the regimen prescribed. Route of administration had no influence on risk estimates. The Million Women Study Investigators concluded that HRT use was probably responsible for an extra 20000 breast cancer cases in the UK over the last 10 years. Similar and equally speculative estimates suggest that postmenopausal obesity has probably accounted for 50000 extra breast cancers and alcohol intake in women aged between 45 and 64 years at least 16000 additional breast cancers over the same period.

The risk estimates reported with unopposed and combined HRT in the Million Women Study are greater than those reported by the WHI study investigators. Although the Million Women Study is large, it is observational and potentially open to confounding. If differences exist between women attending the NHSBSP and those who do not, and between attendees who agreed or declined to participate in the study, this could bias the reported results. It should therefore be interpreted in the context of completed/ongoing placebo-controlled HRT trials. There is also discrepancy with the WHI study, where an increase in breast cancer risk did not begin to emerge until 3 years after randomisation. This contrasts with the Million Women Study where risk was reported to increase after less than 1 year's exposure. A likely explanation is that the Million Women Study Collaborators underestimated the total duration of HRT exposure, as all the risk estimates were based on the baseline questionnaire data. If women continued using HRT and only stopped at cancer diagnosis this means that an average of 1.2 years (i.e. the mean time to cancer diagnosis from initial mammography) should be added to each of the duration categories for current HRT users. This shifts the pattern of risk with time to one that is more consistent with the WHI study. The Million Women Study is the first to provide any epidemiological data about tibolone and breast cancer risk, although numbers are relatively small and the potential for confounding exists. The increased risk reported conflicts with the hypothesis that as tibolone does not increase postmenopausal mammographic breast density, it is unlikely to have a significant stimulatory effect on the breast. $^{7}$

Breast cancer incidence is a relevant outcome but breast cancer mortality is the most important endpoint. With the exception of the Million Women Study, which has reported an increased risk, albeit of borderline statistical 
significance, in breast cancer mortality for current HRT users at baseline (RR 1.22; 95\% CI 1.00-1.48), other observational evidence has not shown a similar effect. $5,8,9$ The increased mortality reported by the Million Women Study Collaborators does not necessarily imply that HRTassociated tumours have to be more advanced, which would contradict previous observational evidence. Instead, the increased mortality could simply reflect the fact that more tumours were diagnosed in women with a history of HRT exposure. The number of breast cancer deaths in the Million Women Study $(\mathrm{n}=637)$ is too small for relationships between mortality and the type and duration of HRT use to be estimated, and without any information on the phenotype, stage or treatment of incident breast cancers, the data cannot be interpreted. Despite the large number of women randomised in the WHI study, only eight breast cancer deaths were reported, four in each of the HRT and placebo groups. The WHI study reported that HRTassociated tumours were larger (mean size difference of $2 \mathrm{~mm}, \mathrm{p}=0.04$ ) and more likely to be node-positive [proportion of positive nodes with HRT $(\mathrm{n}=25) 25.9 \%$; 95\% CI 19.5-30; placebo $(\mathrm{n}=21) 15.8 \%$; 95\% CI $10-23.1]$. This trend is of borderline significance $(\mathrm{p}=0.08)$ and the wide, overlapping confidence intervals raise uncertainty as to whether additional breast cancer events would weaken or strengthen this association. There was no difference between treatment groups with respect to tumour grade, histological type and hormone receptor positivity, but the WHI study is almost certainly too small to detect such differences. Most postmenopausal tumours are ER+ve $(87 \%)$ and invasive lobular cancers constitute only about $10 \%$ of all invasive disease. Thus, approximately 1000 breast cancer events would be needed to detect a $5 \%$ difference compared with placebo, but there were only 349 invasive cancers in the latest WHI report. Calculation of prognosis with the validated and widely used Nottingham Prognostic Index does not place HRTassociated cancers in a worse prognostic group compared with placebo. The difference in estimated 10-year survival based on mean tumour size, a mean tumour grade of 2 and weighted according to the proportion of node-positive disease is very small (i.e. $62.5 \%$ for HRT versus $61 \%$ for placebo). ${ }^{10}$ For symptomatic breast cancer survivors treated with short-term (a median of 30 months) unopposed or combined HRT no increase in recurrence has been reported from observational studies. Large-scale randomised trials are now underway. ${ }^{11,12}$

Studies evaluating the effect of HRT on breast epithelial proliferation and mammographic density provide further support for a positive association between combined HRT and an increased risk of breast cancer. The placebocontrolled Postmenopausal Estrogen/Progestin Interventions (PEPI) Trial demonstrated that unopposed oestrogen (i.e. CEE $0.625 \mathrm{mg}$ ) does not increase mammographic breast density, whereas approximately $25 \%$ of women allocated to combined HRT containing MPA are likely to develop an increase in mammographic density within the first year of use, irrespective of whether the MPA administration is cyclical or continuous. ${ }^{13}$ Continuous combined HRT (i.e. CEE $0.625 \mathrm{mg}$ plus MPA $2.5 \mathrm{mg}$ ) has also been reported to increase the number and size of proliferating breast epithelial cells in tissue biopsies from areas of abnormal mammographic breast density compared with biopsies from women with no history of HRT exposure, or those who are taking unopposed oestrogen. ${ }^{14}$

The WHI study reported a statistically significant increase in the number of abnormal mammograms in women allocated to HRT compared with placebo $(\mathrm{p}<0.01)$ from the first year of follow-up. ${ }^{4}$ However, the absence of details about screening sensitivity, specificity and quality assurance within the WHI study (women were screened at over 3000 centres), combined with the fact that no established national or regional screening programmes perform annual, two-view mammography (the screening method utilised in WHI), makes it difficult to extrapolate the results to the performance of other screening programmes. About half $(53 \%)$ of the $33 \%$ of women attending the NHSBSP currently use combined HRT. ${ }^{6}$ In view of the PEPI trial results the likely proportion expected to develop any density increase would be approximately $5 \% .13$

\section{Hormonal contraceptives and breast cancer risk}

No large-scale, randomised trials of hormonal contraceptives have been conducted and advice is therefore based on observational studies that have predominantly evaluated the impact of combined oral contraceptives (COCs). The Collaborative Group for Hormonal Factors in Breast Cancer re-analysis of worldwide epidemiological studies (1996) reported a small increase in the risk of developing breast cancer with current use of COCs (RR $1.24 ; 95 \%$ CI $1.15-1.33$ ), with no excess risk 10 years after cessation. ${ }^{15}$ Although risk is greater in women commencing use in their teens, this has little effect on the estimated cumulative breast cancer incidence because of the low baseline risk at this age. As breast cancer incidence increases with age, excess risk is probably determined by the age at last exposure to COCs rather than age at commencing use or duration of exposure. Tumours developing in COC users are less likely to have spread beyond the breast at presentation compared with non-users (i.e. RR 0.88 ; 95\% CI 0.81-0.95) although early detection bias cannot be excluded. ${ }^{15}$ The Collaborative re-analysis found no difference in breast cancer risk according to specific types of oestrogen or progestogen although a reduced risk has been reported with lower dose combined preparations. ${ }^{16}$ Differentiation of risk according to progestogen type is limited due to lack of statistical power but overall there is no apparent difference in risk across progestogens used in current prescriptions. ${ }^{16,17}$ Depot progestogens and progestogen-only pills (POPs) have not been shown to increase risk significantly but conclusions are limited by the small number of events upon which risk estimates are based. ${ }^{15,17}$

Whether family history modifies the effect of hormonal contraception on breast cancer risk in young women is an important but unanswered question. The Collaborative reanalysis reported similar risk estimates for breast cancer irrespective of family history (defined as one or more affected first-degree relatives) but estimates were based on all ages combined. ${ }^{15}$ For women with BRCA1 or BRCA2 gene mutations the effect of COCs on breast cancer risk is contradictory but has to be weighted against the potential benefit of a reduction in the incidence of ovarian cancer. ${ }^{18,19}$ No data are available regarding the use of progestogen-only contraceptives.

COCs have been shown to increase breast epithelial proliferation. ${ }^{20}$ Whilst proliferation does not appear to differ according to the progestogen (i.e. comparing levonorgestrel with desogestrel), a positive correlation between increasing serum levonorgestrel and proliferative indices has been reported. ${ }^{17}$ Evidence about the effect of POPs, depot progestogens or progestogen-releasing intrauterine systems (IUSs) on epithelial proliferation is lacking. If proliferation is determined by serum progestogen levels, the particularly low levonorgestrel concentrations in users of the Mirena ${ }^{\circledR}$ IUS (i.e. $~ 0.5$ $\mathrm{nmol} / \mathrm{l}$ ) may not have a significant effect, although this requires confirmation. ${ }^{21}$ Studies evaluating the effect of COCs on mammographic density are contradictory. ${ }^{22}$ 
HRT, hormonal contraception and benign breast disease Debate exists as to whether the malignant transformation of normal breast epithelium involves an invariable progression through benign breast change with hyperplasia, atypia and in situ disease, or whether some invasive cancers arise directly from morphologically normal cells. HRT reverses the physiological changes of breast involution and therefore benign conditions such as fibroadenoma, cysts and mastalgia may persist in the menopause with its use. The only study to classify benign histological change accurately according to subsequent breast cancer risk failed to show any adverse effect of oestrogen replacement therapy in women with atypia (which itself confers a five-fold increase in risk), but this estimate was based on only seven breast cancer cases. ${ }^{23}$ However, in a large randomised trial, Tamoxifen ${ }^{\circledR}$ has been reported to reduce breast cancer risk significantly in women with atypical ductal hyperplasia, suggesting indirectly that HRT may have an adverse effect. ${ }^{24}$ The WHI study did not find a significant increase in the risk of ductal carcinoma in situ (DCIS) in women allocated to HRT but as the incidence of DCIS is only about one-fifth of that of invasive cancer in screened populations, the study was probably underpowered to detect any difference. The effect of hormonal contraceptives on benign disease (with or without atypia) and in situ disease is unclear. Risk estimates are based on very small sample sizes and, for benign disease, most studies have utilised histological classifications no longer used in clinical practice. ${ }^{25,26}$

\section{Summary}

In summary, clinical studies evaluating HRT and hormonal contraceptives support the hypothesis that in combination with oestrogen, exogenous progestogens exert a mitogenic effect on the breast. The effects of different progestogens on human breast tissue are unclear due to lack of statistical power, and this limits firm recommendations for clinical practice. For women using HRT and COCs, however, the risk of breast cancer is small and any probable impact on long-term survival is likely to be very small.

\section{Statements on funding and competing interests}

Funding. Jo Marsden is the joint principal investigator for the national UK randomised trial of HRT in symptomatic women with early stage breast cancer and has been sponsored to attend conferences and received speaker's fees from Organon, Orion, Schering, Servier, Solvay Healthcare Ltd and Wyeth. Consultancy fees have been received from Wyeth and Organon and fees for preparation of educational material from Novartis. Competing interests. None identified.

\section{References}

1 Early Breast Cancer Trialists' Collaborative Group. Tamoxifen for early breast cancer: an overview of the randomised trials. Lancet 1998; 351: 1451-1467.

2 Writing Group for the Women's Health Initiative Investigators. Risks and benefits of estrogen plus progestin in healthy postmenopausal women. JAMA 2002; 288: 321-333.

3 Women's Health Initiative (WHI) HRT Update 2002 www.nhlbi.nih.gov

4 Chlebowski RT, Hendrix SL, Langer RD, et al. for the WHI
Investigators. Influence of estrogen plus progestin on breast cancer and mammography in healthy postmenopausal women. The Women's Health Initiative Randomised Trial. JAMA 2003; 289: 3243-3253.

5 Million Women Study Collaborators. Breast cancer and hormonereplacement therapy in the Million Women Study. Lancet 2003; 362: 419-427.

6 Million Women Study Collaborators. Patterns of use of hormone replacement therapy in one million women in Britain, 1996-2000. Br J Obstet Gynaecol 2002; 109: 1319-1330.

7 Lundström E, Christow A, Kersemaekers W, et al. Effects of tibolone and continuous combined hormone replacement therapy on mammographic breast density. Am J Obstet Gynecol 2002; 186: 717-722.

8 Collaborative Group on Hormonal Factors for Breast Cancer. Breast cancer and hormone replacement therapy: collaborative reanalysis from 51 individual epidemiological studies. Lancet 1997; 350: 1047-1060.

9 Nanda K, Bastian LA, Schultz K. Hormone replacement therapy and the risk of death from breast cancer: a systematic review. Am J Obstet Gynecol 2002; 186: 325-334.

10 Kollias J, Murphy CA, Elston CW, et al. The prognosis of small primary breast cancers. Eur J Cancer 1999; 35: 908-912.

11 Col NF, Kirota LK, Orr RK, et al. Hormone replacement therapy after breast cancer: a systematic review and quantitative assessment of risk. J Clin Oncol 2001; 19: 2357-2363.

12 Marsden J, Sacks NPM, Baum M, et al. Are randomised trials of hormone replacement therapy in symptomatic breast cancer patients feasible? Fert Steril 2000; 73: 292-299.

13 Greendale GA, Reboussin BA, Sie A, et al. Effects of estrogen and estrogen-progestin on mammographic parenchymal density. Ann Intern Med 1999; 130: 262-269.

14 Hofseth LJ, Raafat Am, Osuch JR, et al. Hormone replacement therapy with estrogen or estrogen plus medroxyprogesterone acetate is associated with increased epithelial proliferation in the normal postmenopausal breast. J Clin Endocrinol Metab 1999; 84: 4559-4565.

15 Collaborative Group on Hormonal Factors in Breast Cancer. Breast cancer and hormonal contraceptives: collaborative reanalysis of individual data on 53,297 women with and 100,239 women without breast cancer from 54 epidemiological studies. Lancet 1996; 347: 1713-1727.

16 Althuis MD, Brogan DR, Coates RJ, et al. Hormonal content and potency of oral contraceptives and breast cancer risk among young women. Br J Cancer 2003; 88: 50-57.

17 Dumeaux V, Alsaker E, Lund E. Breast cancer and specific types of oral contraceptives: a large Norwegian cohort study. Int J Cancer 2003; 105: 844-850.

18 Narod SA, Dube MP, Klijn J, et al. Oral contraceptives and the risk of breast cancer in BRCA1 and BRCA2 mutation carriers. J Natl Cancer Inst 2002; 94: 1773-1779.

19 Narod SA, Risch H, Moslehi R, et al. Oral contraceptives and the risk of hereditary ovarian cancer. $N$ Engl J Med 1998; 339: 424-428.

20 Isaksson E, von Scholtz E, Odlind V, et al. Effects of oral contraceptives on breast epithelial proliferation. Breast Cancer Res Treat 2001; 65: 163-169.

21 Luukkainen T, Lähteenmäki P, Toivonen J. Levonorgestrel-releasing intrauterine device. Ann Med 1990; 22: 85-90.

22 Gram IT, Funkhouser E, Nordgård L, et al. Oral contraceptive use and mammographic patterns. Eur J Cancer Prev 2002; 11: 265-270.

23 Dupont WD, Page DL, Parl FF, et al. Estrogen replacement therapy in women with a history of proliferative breast disease. Cancer 1999; 85: $1277-1283$.

24 Cuzick J, Powles T, Veronsesi U, et al. Overview of the main outcomes in breast cancer prevention trials. Lancet 2003; 361: 296-300.

25 Rohan TE, Miller AB. A cohort study of oral contraceptive use and risk of benign breast disease. Int J Cancer 1999; 82: 191-196.

26 Friedenreich CM, Bryant HE, Alexander F, et al. Risk factors for benign proliferative breast disease. Int J Epidemiol 2000; 29: 637-644.

The latest version of Notes for Contributors can be found on the Faculty website at www.ffprhc.org.uk. The electronic notes are reviewed quarterly and updated as required. They are published in print in the January edition and in other editions if space allows (see page 232 of this issue). 